\title{
IDEAS PREVIAS Y CAMBIO CONCEPTUAL EN ASTRONOMÍA. UN ESTUDIO CON MAESTROS DE PRIMARIA SOBRE EL DÍA Y LA NOCHE, LAS ESTACIONES Y LAS FASES DE LA LUNA
}

CAMINO, N. Grupo Esquel de Educación en Ciencias. Consejo de Investigaciones, Universidad Nacional de la Patagonia. Ciudad Universitaria.9200 Esquel. Argentina.

\section{SUMMARY}

In this paper we present an experience developed with primary teachers which led to the design of a course, from a constructivistic point of view, on those concepts related to Astronomy included in primary curricula (day and night, seasons, moon phases).

With the comparison of pre/post diagnoses (written questions and clinical interviews), we discuss the effectiveness of this conceptual change strategy and make some conclusions, particularly on teacher training.

\section{INTRODUCCIÓN}

El área de ciencias naturales en los profesorados de formación básica para maestros de nivel primario en la Argentina no ha logrado aún tun desarrolio riguroso y profundo de sus contenidos conceptuales específicos.

Sin embargo, las ciencias naturales aparecen como un área importante en el currículo del nivel primario, lo que trae aparejado serias dificultades a los maestros debido a que no tienen formación específica en el área y la bibliograffa disponible en nuestro medio, acorde con la didáctica actual, es muy escasa. Además, todos conocemos cuán interesados e incisivos están los chicos por los fenómenos del mundo natural, lo que implica un requerimiento aun mayor hacia los docentes. Estas consideraciones son particularmente ciertas para los conceptos relacionados con astronomía.

Finalmente, el constructivismo, en general, se ha desarrollado con más fuerza en la lectoescritura y en matemática, siendo su inserción en el área de ciencias naturales bastante pobre.
Elegimos, entonces, trabajar con maestros, desde una concepción constructivista, aquellos conceptos de astronomía que tuvieran mayor inserción en el currículo de primaria, que se hicieran evidentes en fenómenos cotidianos y que nos permitieran realizar experiencias con materiales de bajo costo que brindaran elementos a los maestros para comenzar su camino en el cambio conceptual. Por estas razones los temas elegidos fueron el día y la noche, las estaciones y las fases de la Luna.

\section{DESCRIPCIÓN DEL ESTUDIO}

Este trabajo es la continuación y ampliación de uno anterior (Camino 1991) en el que realizamos una comparación entre las ideas previas que estudiantes de magisterio de Esquel y Tasmania (Jones 1988) tenían sobre estos mismos conceptos. En aquella oportunidad pudimos comprender, mediante un extenso diagnóstico, las 
ideas previas existentes en los adultos que participaron de la experiencia en relación con los tres fenómenos estudiados.

Para este nuevo estudio, nuestra pregunta básica fue, si mediante éste (Driver 1978, 1981, 1986, 1986 y 1989, Gilbert 1983, Hewson 1989, Novack 1988, Osborne 1983 y 1989, Pope 1983, Posner [982, Sebastià 1989 y Zylberstajn 1985), era posible brindar elementos a los docentes que participaran en los mismos para que modificaran en alguna medida el conjunto de ideas previas que ya tenían.

\section{Diseño de la unidad didáctica}

Así, diseñamos tal unidad didáctica de modo que contemplara fundamentalmente dos aspectos:

a) el conjunto de los docentes participantes;

b) La secuencia conceptual que, a nuestro criterio, es la que mejor se adapta al propósito buscado.

La respuesta a la pregunta de partida se construiría mediante la evaluación del desarrollo de los talleres y de la comparación entre los diagnósticos antes (pre) y después (post) de la implementación de la unidad didáctica.

\section{Ideas previas existentes}

El estudio de 1991 y la literatura disponible contribuyeron al presente trabajo al considerarlos como un diagnóstico base, que fue ajustado a cada grupo particular de docentes realizando diagnósticos de pre implementación de la unidad didáctica.

Tal diagnóstico comprendía dos partes: por un lado, una pregunta escrita abierta, administrada a todos los docentes, la cual requería responder sobre las causas de estos fenómenos, solicitando explícitamente la realización de dibujos; y, por otro lado, entrevistas unipersonales deI tipo piagetianas no estructuradas (Ault 1988, Bleger 1985, Erickson 1979, Linder 1989, Moreira 1989 , Nahoum 1961, Nussbaum 1976, Piaget 1984, Pines 1978 y Posner 1982), de carácter voluntario, las cuales fueron audiograbadas y transcriptas en su totalidad. Con este diagnóstico pre se elaboraron los modelos de explicación que se presentan en las figuras 1, 2 y 3 (los dibujos son originales realizados por los maestros participantes)

El diagnóstico post tuvo una estructura similar: la parte escrita se administró a todos los docentes a quienes se entregó una hoja por cada fenómeno en la que constaban los modelos de explicación que resultaron del citado estudio de 1991. Se les solicitaba que eligieran uno o varios de ellos, de modo que la explicación quedara totalmente completa. Las entrevistas tuvieron las mismas características que en el diagnóstico de pre implementación.

\section{Secuencia conceptual}

De acuerdo con nuestra experiencia y criterio como docentes en esta área, elegimos desarrollar los conceptos inherentes al día y la noche, las estaciones y las fases, desde tres ejes, interrelacionados en forma dinámica durante el desarrollo de los talleres, aportando los elementos necesarios para la comprensión completa de los tres fenómenos. Tales ejes fueron naturalmente optimizándose en su estructura y relaciones a través de la práctica y de la reftexión producidas en los tres años que duró esta experiencia. Éstos son:

- Conceptos físicos básicos:

a) el movimiento

b) la energía

- Relaciones espaciales:

a) las dimensiones

b) Ia ubicación relativa del observador

- La observación:

a) el sujeto que observa

En el desarrollo de estos ejes tuvimos especial cuidado en no presentarlos como válidos sólo para el sistema Tierra-Sol-Luna sino para todo el universo. Con esto buscábamos ampliar nociones detectadas, un tanto antropocéntricas, como: «sólo la Tierra tiene gravedad», «no sé si los planetas tienen estaciones»; o como las dos citas siguientes:

I: ¿Tendrá día y noche Marte?

E: Si tiene un Sol, sí.

Beatriz, 40 años, pre.

I: ¿Y qué les produce [a los pianetas] estar más cerca o más lejos del Sol?

E: Marte creo que está más cerca $[. .$.$] entonces una temperatura$ mucho mayor que la Tierra[...] o sea [...] la Tierra creo que estaría casi en el medio [...] por eso la temperatura es casi ideal para que la gente viva o haya vida, creo [...] sino en otros planetas habría vida.

Ivanna, 21 años, pre.

El tratamiento en particular de los tres fenómenos bajo estudio tuvo los siguientes momentos:

a) Descripción de lo observable cotidianamente desde un determinado lugar geográfico.

b) Explicación astronómica, cuidando de que la matemática necesaria fuera reducida al máximo posible (proporciones y representación gráfica) y que el uso del pizarrón fuera como apoyatura o síntesis de lo realizado observacionalmente o con material concreto.

c) Reinterpretación de to observado cotidianamente a la luz de la explicación astronómica. 
Figura 1

Modelos utilizados para explicar el día y la noche.

\section{Modelo I}

La Tierra orbita en torno al Sol quedando mitad iluminada y mitad en oscuridad; a su vez rota sobre su eje en veinticuatro horas lo que produce que los puntos sobre su superficie queden alternativamente en la porción iluminada (día) y en la porción en oscuridad (noche). Puede indicarse la inclinación real del eje de la Tierra o cualquier otra.

\section{Modelo 2}

La Tierra orbita al Sol en veinticuatro horas, sin girar sobre su eje.

\section{Modelo 3}

La Tierra rota sobre su eje sin trasiadarse, ubicada en el centro de la Luna y el Sol, diametralmente opuestos, los que no rotan ni se trasladan.

Al girar la Tierra, se ve al Sol de día y a la Luna de noche. Puede indicarse cualquier inclinación del eje terrestre.

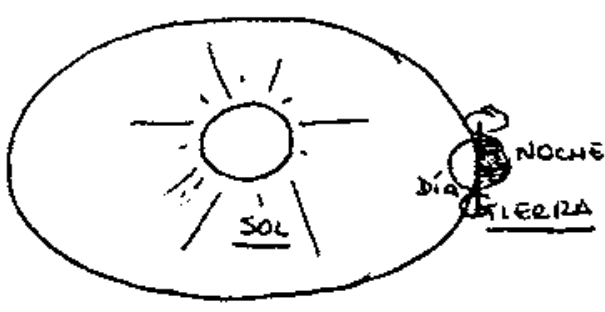

(sin dibujo original)

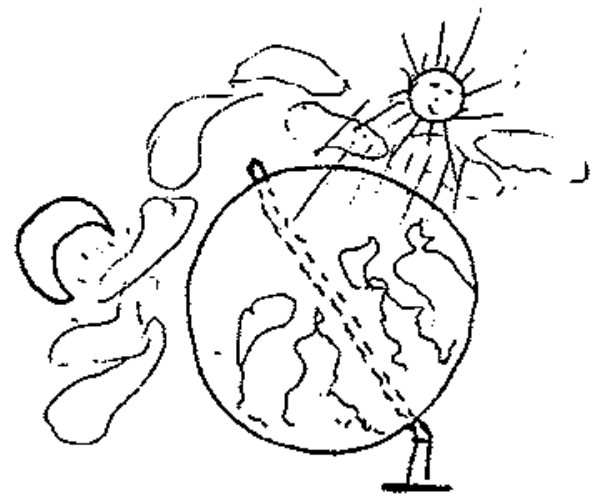

\section{Modelo 4}

La Tierra está en reposo, ubicada en el centro de la Luna y el Sol, diametralmente opuestos, los que orbitan en torno a ella en veinticuatro horas, por lo que se ve al Sol de dia y a la Luna de noche.

Puede no hacerse referencia a la Luna.

Puede indicarse cualquier inclinación del eje terrestre.

\section{Modelo 5}

(sin dibujo originai)

Explicaciones muy vagas que indican que «el Sol es tapado por la sombra de la Luna sobre la Tierra» o que «la Luna tapa al Sol» para producir la noche.

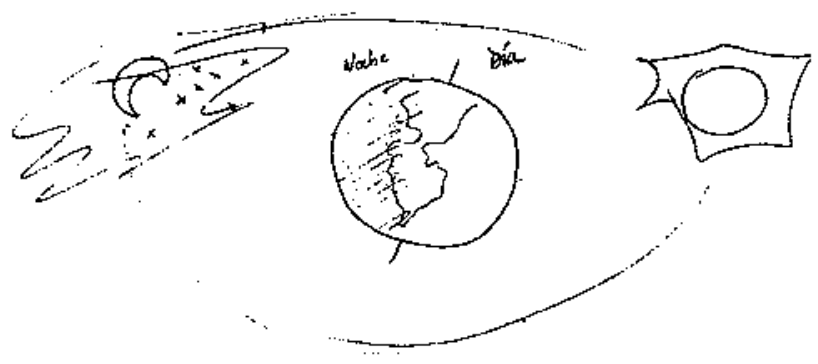


d) Discusión de las actividades realizadas intentando concienciar las contradicciones que pudieran existir con las ideas previas diagnosticadas o intentando fortalecer aquellas ideas previas que estuvieran de acuerdo con el desarrollo conceptual propuesto.

\section{Conceptos físicos básicos}

Los conceptos aquí inciuidos han sido tratados de forma permanente durante el desarrollo de los talleres, volviendo sobre ellos cuando era necesario para profundizar o aclarar algún aspecto particular del día y la noche, las estaciones o las fases. No hubo ningtuna actividad específica diseñada para tratar el movimiento o la energía pero, por su naturaleza, evidentemente estuvieron incluidos de algún modo en las actividades principales llevadas a cabo.

\section{Movimiento}

«El Sol no se mueve, está quieto»; «la L una no gira sobre sí misma, por eso es que siempre le vemos la misma cara»; "no sé si los demás planetas tendrán rotación» y diversas afirmaciones como éstas indican que la noción de movimiento no está, en general, bien comprendida y ni siquiera considerada como una situación común a todo lo existente en el universo.

La estructura conceptual que elegimos fue la siguiente:

-- Todos los cuerpos existentes en el universo se mueven.

Todos giran sobre sí mismos y todos se trasladan en relación a otro cuerpo.

- Estos dos estados de movimiento son independientes el uno del otro.

- El girar sobre sí mismo depende de las condiciones iniciales de formación del cuerpo (no depende ni de su tamaño ni de la distancia a la que se traslada en torno a otro cuerpo).

- El trasladarse en torno a otro cuerpo está determinado por interacción gravitatoria.

En particular se discutió de forma extensa -utilizando materiales concretos y dramatizaciones- el movimiento de la Luna, demostrando la razón de que aun girando sobre su propio eje enfrente a la Tierra siempre la misma mitad de su superficie.

\section{Energia}

Podríamos considerar el día y la noche, las estactones y las fases como fenómenos sencílos desde un punto de vista astronómico; sin embargo, la dificultad para comprenderios en profundidad radica en que para ello es necesario antes comprender conceptos como, por ejemplo, energía, calor y temperatura, luz, etc. No es nuestra intención profundizar aquí sobre las ideas previas y su tratamiento en esas áreas, ampliamente desarrollados en la literatura; sólo queremos mencionar que es posible identificar tales ideas en las explicaciones más comunes en relación con los tres fenómenos citados.

El desarrollo conceptual que elegimos aquí fue plantear una transferencia de energía entre un sistema a gran temperatura (el Sol) -presentando de forma sencilla el mecanismo de fusion nuclear, ya que la idea de que el Sol es una bola de fuego es compartida por casi todos los docentes participantes-, y un sistema a baja temperatura (la Tierra, la Luna, los planetas, etc.).

Los procesos en juego para tal transferencia son los que llamamos Iuz y calor, actuando ambos en forma simultánea.

Por estar orbitando en torno al Sol, cualquier cuerpo, planeta o satélite, tendrá iluminada una mitad y oscura la otra mitad; $y$, por estar girando sobre sí mismo, los distintos puntos de su superficie estarán cierto tiempoen la mitad iluminada y cierto tiempo en la mitad oscura. Llamamos a esto respectivamente día y noche.

La intensidad de energía recibida por el planeta como un todo dependerá de la forma (excentricidad) de su órbita y del tamaño de la misma: a igual excentricidad recibirán menos energía los planetas más lejanos; y en una órbita de gran excentricidad, cuando el planeta se encuentra en la parte más cercana al Sol, recibe más energfa que cuando se encuentra en la parte más lejana.

El promedio de la energía recibida en los distintos lugares sobre la superficie del planeta dependerá de la ubicación de los mismos en relación con la dirección al Sol, lo cual estará determinado por la orientación del eje de rotación del planeta respecto del eje de la órbita del mismo en relación con el Sol. Esto en definitiva es lo que determina, en cada punto del planeta, el ángulo de incidencia de los rayos solares, la altura del Sol sobre el horizonte, la relación luz-oscuridad dentro de un período de rotación (duración del día y de la noche) y la temperatura media en cada época del año, características éstas que irán variando pertódicamente debido al movimiento del planeta en su órbita; llamamos a esto estaciones.

\section{Relaciones espaciales}

Los dos aspectos que siguen han sido tratados tanto en forma permanente como a través de actividades concretas, según se describe a continuación.

\section{Dimensiones}

Hemos encontrado que la imagen del sistema TierraSol-Luna y también del sistema solar y del universo en general, tanto en tamaños como en distancias y tiempos, es muy limitada.

Pot esta razón, desarrollamos dos actividades: una consistió en la representación a escala del sistema TierraSol-Luna, en distancias, tamaños y forma de las órbitas, 
discutićndose también la cscala real del sistema solar como un todo; la segunda actividad fue medir mediante una cámara oscura el diámetro real del Sol y de la Luna. Ambas actividades fucron tomadas y modificadas del Project STAR (1993).

Se discutió también de forma extensa que cl modelo $2 \mathrm{a}$ para explicar estaciones (por una gran excentricidad de la órbita terrestre) no es adecuado para la Tierra, pero, por ejempio, sí para un cometa.

Aquí planteamos que, desde nuestro punto de vista y pensando en el nivel primario, es más conveniente, y no se cae en errores conceptuales graves, presentar las orbitas planetarias - no las de los cometas-directamente como circunferencias; esto es debido a que consideramos que presentarlas como elipses, tal como aparece en la mayoría de los textos, sin hacer una discusión del parámetro excentricidad, trae como consecuencia la idea de largas elipses para todos los planetas con el consecuente modelo 2 a para la explicación de las estaciones.

\section{Ubicación relativa del observador}

I: Cuando yo veo cuarto creciente, ¿una persona en China qué vería?

E: Lo contrario, cuarto menguante.

Beatriz, 40 años, pre.
El día y la noche, las estaciones y las fases serían fenómenos fácilmente explicables, para todos, si pudiéramos tener una perspectiva desde fuera del sistema Tierra-Sol-Luna. Consideramos que la dificultad no radica en los fenómenos en sí mismos sino en que buscamos comprenderlos desde una perspectiva topocéntrica, sin generar herramientas que nos permitan imaginar otros puntos de vista.

El fenómeno de las fases en especial fue presentado como la forma en que se ven el día y la noche y las estaciones de un determinado planeta, si al mismo se obscrva desde un lugar exterior a él. Por ejemplo, analizamos de forma extensa si desde la Luna se verían fases en la Tierra y cómo serian en relación con las fases que descle la Tierra vemos en la Luna, o también si es posible ver fases en Plutón o en Venus, o...

A simismo, trabajamos sobre la idea de que las fases son sólo la apariencia de un planeta en relación con sus zonas bajo Iuz u oscuridad, y que no tienen relación alguna con el relieve que pueda verse del mismo; por ejemplo, Venus tiene fases visto desde la Tierra, pero no es posible ver su relieve.

Modelos concretos (Camino 1993), dramatizaciones y diagramas, como, por ejemplo, el que aparece en la figura 4 (en total se utilizaron ocho esquemas como el de la figura, según las distintas posiciones de la Luna en su órbita), fueron las herramientas utilizadas para producir

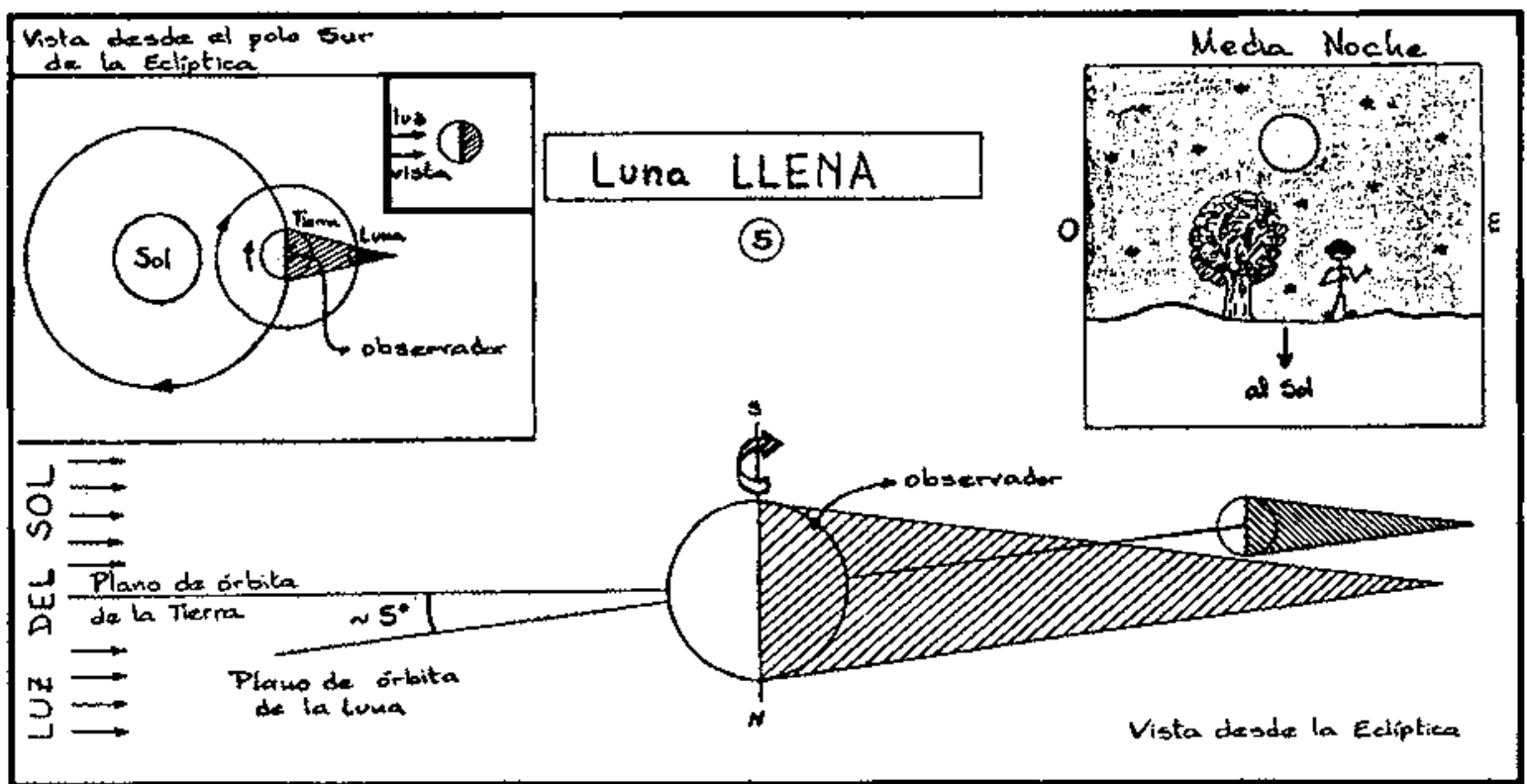


este cambio en la perspectiva con la que analizamos estos fenómenos.

\section{Observación}

\section{El sujeto que observa}

Consideramos que la vida cotidiana en la sociedad actual nos ha llevado paulatinamente a perder nuestra capacidad de observación, en particular la del mundo físico en el que vivimos. En to que a la educación en ciencias se refiere, esto tiene implicaciones muy serias, ya que en general no nos motivan los fenómenos naturales. Nuestro interés está dirigido hacia actividades de fácil gratificación, de tipo consumista, y cuando buscamos, como docentes, generar cierto tipo de reflexión en los alumnos utilizando la observación crítica, se levanta una barrera que parece ser infranqueable.

Por esto, buscamos fortalecer la capacidad de observación y de análisis crítico de los docentes participantes a traves de diversas actividades:

Una de esas actividades requerfa realizar un dibujo de «cómo es Esquel desde arriba» (Camino 1993). Esta actividad fue la que abrió los talleres, permitiéndonos contextuar nuestra concepción de la tarea educativa. Los distintos puntos de vista de cada uno de los docentes participantes ante una "realidad objetiva», como podría ser la ciudad en la que todos vivían, evidenció lo que ocurre dentro de las aulas, en todos los aspectos, con las implicaciones que ello tiene cn cuanto a evaluación, ideas previas, respeto por las concepciones y creencias de los demás, etc.

- Otra actividad fue la observación de la Luna durante por lo menos un mes, -realizando gráficos de su posición y apariencia-, Ia cual fue adaptada, principalmente, de B. Iones (1990), del Project STAR (1993) y de N. Lanciano (1989).

- La última actividad fue la observación sistemática de la sombra de un gnomon, adaptada, principalmente, del Project STAR (1993), de N. Lanciano (1989) y de L. Viglietta (1991).

\section{Implementación de la unidad dídáctica}

Buscamos seguir en la implementación de la unidad didáctica los pasos recomendados en la literatura según la perspectiva constructivista de cambio conceptual.

En este sentido hemos intentado que cada maestro fuera activo participante en el desarrollo y reflexión de las actividades, demostraciones y discusiones grupales mantenidas a lo largo de los talleres. Consideramos que la respuesta ha sido satisfactoria en tanto que la elaboración de informes de trabajo creativos y de proyecciones para su adaptación al nivel primario mostraron un com- promiso personal y una evolución cierta de las ideas previas existentes.

\section{Estructura general de los talleres}

Los talleres (denominados «La Tierra, la Luna, el Sol y nosotros") se desarrollaron durante veinticuatro horas de reloj, divididas en reuniones de cuatro horas durante un lapso de unos dos meses.

Las actividades (observación de la Luna, observación de la sombra de un gnomon, medida del diámetro solar y $\mathrm{cl}$ cálculo y representación del sistema Tierra-Sol-Luna a escala) fueron realizadas por los maestros en los períodos entre las reuniones de trabajo.

Los docentes trabajaron en grupos para la realización de las actividades propuestas, presentaron informes escritos; e individualmente en la concreción de un trabajo final, que debía proponer algún tipo de adaptación didáctica al nivel primario de los conceptos trabajados en el taller.

\section{Población comprendida en este estudio}

Los talleres se desarrollaron en 1991 y 1993 en la ciudad de Esquel y en 1992 en la ciudad de El Bolsón.

Los maestros que participaron al menos en alguna de las actividades (ya que existió una deserción considerada normal para este tipo de talleres), fueron ochenta y cinco (85), con edades comprendidas entre los diecisiete (17) años (algunos estudiantes de magisterio), y los cincuenta (50) años, con un ochenta por ciento de ellos $(80 \%)$ menores de treinta y un (31) años. El noventa y cinco por ciento $(95 \%)$, aproximadamente, del total de docentes comprendidos en este estudio fueron mujeres.

Se registraron setenta y cuatro (74) diagnósticos escritos pre y cuarenta y tres (43) diagnósticos escritos post. Se tomaron treinta y cuatro (34) entrevistas pre y catorce (14) entrevistas post.

\section{ANÁLISIS Y EVALUACIÓN DE LOS DIAG- NOSTICOS ESCRITOS PRE/POST}

Se presentan a continuación, mediante una tabla y gráficos de barras, las comparaciones efectuadas entre los diagnósticos escritos pre/post, para cada fenómeno (tabla 1 y figuras 5,6 y 7 ).

En general puede verse una gran disminución de las categorfas unificadas NS (no significativo, o sea, aquellos intentos de respuesta que no tenían significado coherente alguno) y $\mathrm{NC}$ (no contesta). A nuestro entender, esto se debe a dos causas: una de ellas es que efectivamente los docentes tuvieron que contestar, con cierto sentido y seguridad, al diagnóstico post; la otra causa es que, al ser éste del tipo de selección múltiple, sc les presentaba una serie de modelos ya definidos y ellos debían elegir uno o varios para explicar los fenómenos. 
Figura 7

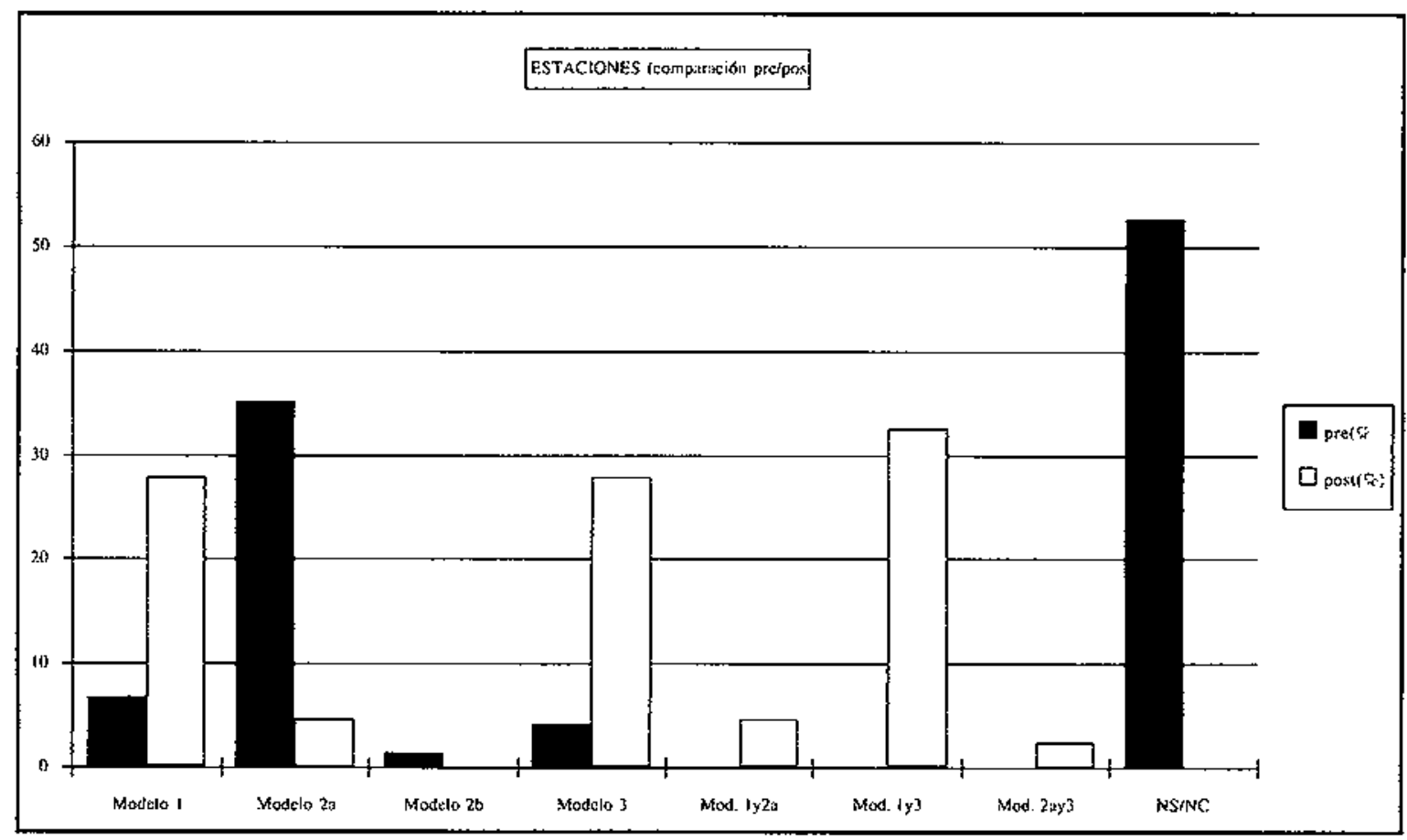

Por lo tanto, es posible que hubierá una cierta tendencia a no dejar de indicar de algún modo al menos una de las opciones.

Es bajo el número de docentes que contestan con modeIos utilizados por chicos o adolescentes, como, por ejemplo, los modelos, 2, 3, 4 y 5 de día y noche, o los modelos 3 y 4 de fases (estas ideas para tales edades han sido estudiadas por diversos investigadores, entre otros, Baxter 1989 y Jones 1987).

\section{En relación con el día y la noche}

Podemos indicar como alto el número de docentes que contestan con el modelo considerado correcto (modelo 1) tanto en el pre como en el post: 53 de 74 (72\%) en el pre y 28 de $43(65 \%)$ en el post. No tenemos una clara noción de por qué aumentan los modelos 3 y 4 en el post.

\section{En relación con las estaciones}

Es evidente el gran crecimiento de las respuestas que utilizan el modelo considerado correcto (modelo 1), ya sea puro o en combinación con otros (l y $2 a$, I y 3 ). El total de docentes que lo usan de alguna manera en el post es 28 sobre $43(66 \%)$, contra 5 sobre $73(7 \%)$ en el pre.

Es notoria la disminución del modelo 2 a (por gran excentricidad), que pasó de 26 sobre 74 (35\%) en el pre, a sólo 5 sobre 43 ( $12 \%$ considerando las combinaciones con los modelos 1 y 3 ).

Consideramos que el gran crecimiento del modelo 3 (por ubicación geográfica), 12 sobre $43(28 \%)$ sin considerar sus combinaciones con los modelos 1 y $2 \mathrm{a}$, puede deberse a que durante el desarrollo de los talleres discutimos extensamente las particularidades de los fenómenos en relación con los distintos puntos de la geografía terrestre, pudiendo no haber quedado bien diferenciado a qué hacíamos referencia al hablar del eje inclinado y de la dirección de cada punto hacia el Sol, elementos que sí fueron incorporados pero no de la manera deseada por nosotros.

\section{En relación con las fases}

Es evidente el gran aumento de las respuestas que utilizaron el modelo considerado correcto (modelo 1) para explicar este fenómeno: 32 sobre 43 (74\%) en el post, contra 6 sobre $74(8 \%)$ en el pre.

El modelo 2 (eclipse lunar), el más común para explicar fases en general, no varió en la comparación pre/post aunque en ambos casos es baja su incidencia en to diagnosticado en este trabajo. Aumentaron, aunque de manera no muy importante, los modelos 3 y 4 ; el aumento en el modelo 3 puede deberse a que mencionamos que esta explicación no es la correcta para el fenómeno de las fases en sí mismo, pero sí explica que, cuando recién 
Tabla I

\begin{tabular}{|c|c|c|c|c|}
\hline & pre(cant) & post (cant) & $\operatorname{pre}(\%)$ & post $(\%)$ \\
\hline Modelo & 53 & 28 & 72 & 65 \\
\hline Modelo 2 & 0 & 0 & 0 & 0 \\
\hline Modelo 3 & 0 & 5 & 0 & 12 \\
\hline Modelo 4 & 1 & 3 & 1 & 7 \\
\hline Modelo 5 & 0 & 0 & 0 & 0 \\
\hline Modelo 1 y 3 & 0 & 5 & 0 & 12 \\
\hline Modelo 3 y 4 & 0 & 2 & 0 & 5 \\
\hline NS/NC & 20 & 0 & 27 & 0 \\
\hline Total & 74 & 43 & 100 & 100 \\
\hline \multicolumn{5}{|c|}{ FSTACIONES (pre/post) } \\
\hline & pre(cant) & post(cant) & pre(\%) & $\operatorname{post}(\%)$ \\
\hline Modelo 1 & 5 & 12 & 7 & 28 \\
\hline Modelo 2a & 26 & 2 & 35 & 5 \\
\hline Modelo $2 b$ & 1 & 0 & 1 & 0 \\
\hline Modelo 3 & 3 & 12 & 4 & 28 \\
\hline Modelo 1 y $2 a$ & 0 & 2 & 0 & 5 \\
\hline Modelo 1 y 3 & 0 & 14 & 0 & 33 \\
\hline Modclo $2 a$ y 3 & 0 & 1 & 0 & 2 \\
\hline NS/NC & 39 & 0 & 53 & 0 \\
\hline Total & 74 & 43 & 100 & 100 \\
\hline \multicolumn{5}{|c|}{ FASES (pre/post) } \\
\hline & pre(cant) & post (cant) & pre(\%) & post $(\%)$ \\
\hline Modelo 1 & 6 & 32 & 8 & 74 \\
\hline Modelo 2 & 3 & 1 & 4 & 2 \\
\hline Modelo 3 & 0 & 2 & 0 & $s$ \\
\hline Modelo 4 & 0 & 1 & 0 & 2 \\
\hline Modelo 1 y 2 & 0 & 1 & 0 & 2 \\
\hline Modelo 2 y 3 & 0 & 2 & 0 & 5 \\
\hline Modelo 3 y 4 & 0 & 1 & 0 & 2 \\
\hline Modelo 1 y 4 & 0 & 1 & 0 & 2 \\
\hline $\mathrm{NS} / \mathrm{NC}$ & 65 & 2 & 88 & 5 \\
\hline Total & 74 & 43 & 100 & 100 \\
\hline
\end{tabular}

comienza la fase creciente, el reflejo sobre ta Tierra es lo que permite que veamos la totalidad del disco lunar (desde la Luna se vería una Tierra llena recién comenzando a menguar).

\section{ANÁLISIS Y EVALUACIÓN DE LAS ENTREVISTAS PRE/POST}

Debido al carácter voluntario de las entrevistas y al hecho de ser no estructuradas, no se entrevistó a todos los docentes y al realizar una entrevista no se buscó tratar todos los conceptos estudiados, ni buscar necesariamente la comparación pre/post con la misma persona. Por estas razones, las porciones de entrevistas que presentamos a continuación han sido seleccionadas por ser las más representativas de las ideas diagnosticadas mediante esta metodología.

Los nombres que se indican son falsos, no asf la edad y el sexo. Nuestra comunidad es pequeña y, ya que este trabajo será difundido entre los docentes de la zona, para continuar trabajando en el futuro, hemos elegido por razones éticas que sus identidades estén protegidas.

\section{En relación con el día y la noche}

En general el modelo 1 es el más utilizado, tanto en el pre como en el post, tal como se indicara en el análisis del diagnóstico escrito.

I: Suponete que te vas a otro planeta, ¿habrá día y noche allí? E: Supongo que sí; me parece que no [varía con la distancia] porque en realidad ol Sol, esté el planeta cerca o lejos, igual va a alunibrar una cara, o sea, más bien depende de lo que tarde en girar sobre sí mismo.

Azucena, 28 años, pre.

\section{I: ¿Habrá día y noche en Saturno?}

E: Sí, según a qué distancia esté del Sol, por ahí es imperceptible, no va a ser como el día y la noche acá en la Tierra, por ahí es mucho menor la diferencia entre el día y la noche, que va a haber más o menos luz, no va a ver diferencia en la intensidad de la luz, porque lo que sería para nosotros el día, cuando una cara cle Saturno está mirando al Sol, por ahí la luz liega tan tenue que no se va a notar a cuando no está de día.

Gabriela, 24 años, pre.

Es posible encontrar en algunos casos del diagnóstico pre explicaciones que utilizan los modelos 3 y 5 , no así Ios modelos 2 y 4 . Tales modelos no fueron encontrados en el diagnóstico post. Se transcriben a continuación los citadios modelos pre.

F: Es de noche cuando acá en Esquel da para el lado de la Luna, y [es de día] cuando está de este otro lado, enfrentado por el Sol.

Estela, 23 años, pre (modelo 3).

\section{I: ¿En la Luna tendrán día y noche?}

E: Sf, porque, si la Luna va girando alrededor de la Tierra, cuando está acá sería de noche porque la Tierra taparía la luz., como se vería acá, que acá está de día.

I: ¿La tapa, por qué?

E: Porque la Tierra es más grande que la Luna y taparía la luz. que manda el Sol, entonces no se verfa la luz.

Patricia, 28 años, pre (modelo S).

Hemos encontrado que superpuesta a la explicación de las causas del día y la noche, existe otra explicación 
sobre la duración de los mismos. El período completo, o sea la suma de las dos partes que llamamos día y noche, se explica diciendo que es por la velocidad de rotación del planeta sobre su propio eje, pero esa velocidad a su vez es explicada de tres maneras:

Submodelo a). Determinada por las condiciones iniciales de formación del planeta (considerada la explicación correcta).

Submodelo b). Determinada por el tamaño del planeta.

Submodelo c). Determinada por la distancia al Sol.

Así, es posible ver que, aun definiendo correctamente que la mitad iluminada de un cuerpo es lo que llamamos día y la mitad no iluminada es lo que llamamos noche, muchos docentes piensan que el día dura menos que la noche en aquellos planetas muy alejados del Sol y viceversa, confundiéndolo con la intensidad de la luz recibida por el planeta mientras está siendo iluminado por el Sol, o sea durante su día, y incorporando además comentarios tales como: "porque tarda más la luz en llegar» 0 «su velocidad es menor».

Consideramos que esto responde fundamentalmente a dos causas: una de ellas ya fue mencionada al hablar de las concepciones subyacentes a los fenómenos estudiados, en este caso la luz; la otra causa es el uso del lenguaje cotidiano (Lanciano 1989), ya que hemos encontrado que produce gran confusión llamar «día» al perfodo de rotación de la Tierra y al mismo tiempo a lia parte de ese perfodo en la que hay luz del Sol.

I: ¿Y de qué depende cuánto tiempo tarda un planeta en girar sobre su propio eje?

E: De la velocidad que tenga, es decir, si gira muy rápido, cada día y noche tendrá menos tiempo.

I: ¿Y eso tendrá que ver con la lejanía del Sol, con el tamaño del planeta o con alguna otra cosa?

E: No, creo que tiene que ver con su propio movimiento, es un problema del planeta.

Jorge, 21 años, pre (submodelo a).

I: ¿Como te parece que serían los días de los otros planetas?

E: Plutón pienso que no tendría muchodía porque está sumamente alejado del Sol, y en Venus me imagino el día más largo, porque está mucho más cerca del Sol, pienso que debe recibir mucha más luz..

I: Y con respecto al cambio de fecha, suponete que acá en la Tierra tienen que pasar veinticuatro horas para pasas de un dia al otro, ¿en Venus y Plutón, cómo serán esos días, la duración completa?

E: También depende del tamaño. Ptenso que en Júpiter, por cjemplo, el día đebe ser mucho más [...] debe tardar mucho más tiempo en girar sobre si mismo; en cambio Pluton que es tan chiquitito, debe girar mucho más corto, pienso que depende del tamaño de cada planeta.

María, 24 años, pre (submodelos b y c)
La evolución de estas ideas en el diagnóstico post fue variada:

\section{I: ¿Habría alguna diferencia con el día en Venus?}

E: Sí, yo pienso que es porque, si es más grande o más chico el planeta, dura más o menos.

Ivanna, 21 años, post.

I: Suponete que a la Tierra la cambiás a la orbita de Plutón, ien qué variarian sus días y noches?

E: Variarían en la temperatura, en la fuerza con que recibe la luz.

I: ¿Y en cuanto a su đuración?

E: No, porque el movimiento de la Tierra no depende más que de cómo se formó ese planeta, entonces la velocidad va a ser la misma, de rotación, lo único que va a variar es la intensidad de la luz.

Gabriela, 24 años, post.

\section{En relación con las estaciones}

En términos generales, el diagnóstico por entrevistas sigue lo descrito en relación con el diagnóstico escrito, con una comparación similar entre pre/post. EI modelo I se encuentra habitualmente mezclado con el modelo $2 \mathrm{a}$ o con el modelo 3 . Se transcriben a continuación porciones de entrevistas representativas de ambas instancias.

\section{I: ¿Cómo hacés para explicar las estaciones?}

E: Según la posición de la Tierra o la distancia de la Tierra con respecto al Sol y qué parte de la Tierra es la que muestra más su cara hacia el Sol, será la más calurosa y, al contrario, la que esté măs alejada del Sol o para el otro lado sería la tnás fría. Por ejemplo, éste sería el ecuador [...] entonces, como la distancia del ecuador, de la zona cercana al ecuador [...] son las más [...] no las más cercanas, sino que reciben los rayos en forma más directa del Sol, por eso es la más cálida; en cambio el otro recibe de forma oblicua el rayo del Sol, por eso es más frío. En el verano los rayos son más directos y en los otros menos, de eso variará la temperatura.

I: ¿Y qué cambia de una posición a otra en la órbita?

E: Según en qué posición de la elipse que describe la Tierra alrededor del Sol esté, como que acá es más cerca y acá es más lejos, como la elipse tiene forma alargada.

Beatriz, 40 años, pre (modelo I y modelo $2 \mathrm{a}$ ).

I: ¿Tendrá algo que ver la órbita de un planeta en torno al Sol con si tiene estaciones o no?

E: Sí, porque, por ejemplo, la Tierra, que es lo que más nos importa, ¿no?, tiene una órbita eliptica y tambićn tiene que ver la posición de la Tierra con respecto a su eje imaginario y depende de cómo esté, cerca o lejos del Sol y si esta parte está más alejada que ésta [señala dos puntos sobre la superficie terrestre], ésta va a estar en invierno y ésta en verano.

I: ¿Como hacés para que esté más ccrca o más lejos una parte o la otra? 
E: El hecho de que esté inclinado implica que la parte norte va a estar sensiblemente un poquito más cerca que acá y va a recibir los rayos solares más, qué sé yo, más fuertes.

I: ¿Cuál de las dos causas influye más?

E: La de cercanía o no al Sol.

I: ¿Y la otra qué efecto produce?

E: Determina un poco la división en los dos hemisferios, es decir, qué va a pasar acá al norte y qué va a pasar acá al sur.

Eugenia, 17 años, pre (modelos 1 y 3 ).

\section{I: ¿Como explicás las estaciones?}

E: Era una forma elifptica, y cuanto más se acerca al Sol tenemos primavera y verano y cuanto más se aleja del Sol es el otoño e invierno.

\section{I: ¿Fin otros planetas habrá estaciones también?}

E: Sí, porque todos giran alrededor del Sol, lo que yo no me acuerdo es si tienen la misma forma elíptica, el asunto es que si tienen un círculo perfecto no hay estaciones, pero si tienen una forma elíptica que en un momento se acerca al Sol, tiene que haber por lo menos dos estaciones.

Joaquín, 31 años, pre (modelo 2a).

El modelo 2b, aparece de forma excepcional, es rápidamente abandonado, y sería una explicación ad hoc para algunas situaciones no muy bien comprendidas por quien trata de explicarlas.

I: ¿Por qué pusiste el Sol ahí [fuera de la órbita] y no en otro lugar? ¿Por qué no lo pusiste acá [indicando un dibujo anterior en el que figuraba el Sol dentro de la 6rbita]? ¿Da lo mismo?

E: No sé, lo que pasa es que, de acuerdo con la situación que me planteaste, porque acá vos me preguntaste los movimientos de la Tierra [indica el anterior dibujo] y después me preguntaste las estaciones, entonces a mí me parece que el Sol tiene que estar sobre el planeta. No, no da lo mismo, me parece que no da lo mismo.

Cecilia, 22 años, pre (modelo 2b).

Del análisis post se desprende que muchos docentes incorporaron al modelo 1 para su explicación (Pope 1983):

I: ¿Qué tendría que pasar para que esto de las estaciones no sucediera?

E: La inclinación de la Tierra tendría que ser distinta, básicamente.

I: Y sì la orbita de la Tierra fuese una circunferencia perfecta, ¿seguirfa habiendo estaciones?

E: Sí, porque evidentemente no es una elipse como la que nos imaginábamos, casi no variaría porque la excentricidad no es tanto como para decir que habría un gran cambio.

Magalf, 3 I años, post.
E: Se debe primero a la ubicación del planeta Tierra con respecto al centro, al Sol, después por la posición del eje, eso sería io importante.

I: ¿Y cuál de los dos fenómenos es el que más influye?

E: El segundo, la posición del eje de la tierra.

Eugenia, 17 años, post.

I: Si viniese un visitante de Plutón y te pregunta qué es el invierno, ¿qué le dirías?

E: Una estación del año, que se da por la posición del eje imaginario de la Tierra con el Sol, que se da en distintos hemisferios según el momento de la traslación [...] si el hemisferio sur está mirando hacia el Sol, estaría en verano; si el hemisferio norte en otro momento de la traslación estaría mirando hacia el Sol, sería la posición de verano.

Huenú, 20 años, post.

En otros casos, se produce cierto conf licto que queda sin resolución.

I: ¿Qué es el verano. Cómo lo definirías?

E: Hace más calor porque la Tierra está más cerca del Sol o los rayos están más directos $\{\ldots\}$ el Sol con respecto a nosotros, caen en forma más perpendicular y no tan oblicua.

I: ¿Y cómo relacionás eso con que la Tierra está más cerca del Sol?

E: No sé, antes hablábamos de la elipse y ahora sabemos que la elipse no es tal, la explicación que yo tenía antes era que la Tierriz estaba acá más cerca pero ahora sabemos que es más bien redonda [la órbita]]; así que no sé, antes tenía una explicación porque en el momento en que pasaba cerca de las partes más achatadas de la elipse la distancia era mucho más corta con respecto a las puntas.

Beatriz, 40 años, post.

Y en otros casos no se modificó la forma de explicar las estaciones.

I: ¿Cómo le explicarías a un viajero de Venus las estaciones?

E: Le explicaría que según la distancia que tenga la Tierra del Sol via a sentir más frío o más calor, nosotros giramos y rotamos airededor del Sol y al no ser redonda [la órbita], acá [en la posición más cercana al Sol] vamos a recibir más directamente los rayos del Sol.

Sofía, 33 años, post.

I: ¿Hace falta hablar del eje para explicar las estaciones, o no?

E: No, no hace falta porque siempre se va a producir día y noche, lo que varia el eje es la cantidad de horas de luz.

I: ¿Y para las estaciones?

E: Y para las estaciones tampoco, [lo esencial] es que esté más cerca o más lejos.

Joaquin, 31 años, post. 


\section{En relación con las fases}

Del mismo modo que al analizar estaciones, lo descrito en el diagnóstico escrito se repite en las fases. El modelo 1 aparece descrito de forma no muy clara; el más seguro es el modelo 2, y los modelos 3 y 4 no fueron hallados.

I: Si estás parado en la Tierra, ¿le verías fases a Plutón?

E: No, no creo que pudiera ver fases de Plutón; a esa distancia imposible. Lo que pasa es que para el ángulo que hay, digamos, Ia órbita de la Tierra alrededor del Sol, la distancia es muy grande al lado del radio [de la órbita] de la Tierra, entonces la Tierra prácticamente se podría decir que está en la línea del Sol y Plutón, habría una sola fase, siempre iluminada, no le vería una sombrita, serfa imperceptible.

Marcelo, 29 años, pre (modelo 1).

I: ¿Por qué el Sol no ilumina esa parte de abajo?

E: Porqque está interpuesta la Tierra, es como si tıviéramos una línea acá, de la línea para abajo es oscuridad y de la línea para arriba es luz.

Sofía, 33 años, pre (modelo 2).

I: ¿Se te ocurre si desde la Luna le ves fases a la Tierra?

E: La Luna está al lado de lá Tierra, girando alrededor del Sol, sí, se pueden ver las fases de la Tierra.

I: ¿Y cómo sería una Tierra en cuarto creciente?

E: También como una media Luna.

I: Pero, ¿por qué se produce?

E: Cuando la Luna estuviera interpuesta entre la Tierra y el Sol, allí se vería, cuando se produce el eclipse de Sol, se estaría produciendo acá, [señala la Tierra] se verían las fases de la Tierra, como si fueran las fases de la Luna.

I: Desde la Tierra, ¿qué verías en ese mismo momento?

E: Un eclipse de Sol.

I: Ahora suponete que volvés a la Tierra, cuando vos mirâs a la Luna y la ves en fase, ¿por qué se produjo esa fase?

E: Porque la Tierra está interpuesta entre la Luna y el Sol.

Cardela, 40 años, pre (modelo 2).

También en el análisis de las respuestas post para fases podemos encontrar docentes que han incorporado eI modelo 1 .

I: Si te comunicás con alguien en Júpiter, en el Sol, en Marte, ¿todos coincidirían en que la Luna está en cuarto creciente?

E: No, desde el punto de vista terrícola.

I: ¿Qué es to que varía en los demás?

E: El punto de observación.

I: Y si nos fuésemos al Sol, ¿le verfas fases a los demás planetas?

E: No, le veria la parte iluminada, nada más, siempre. No hay cómo verle la parte oscura, porque soy la fuente de tuz.

Marta, 30 años, post.

I: ¿Vos podrías decir que las fases es un fenómeno absoluto, o sea que para todo el universo la Luna está en cuarto creciente, hoy, por ejemplo?

E: No, para nosotros está yendo a liena, para Júpiter puede ser distinto.

I: ¿Y qué es lo que varía?

$\mathrm{E}: \mathrm{El}$ ángulo de donde yo lo miro.

Magalí, 31 años, post.

En otros casos conviven el modelo 1 con el modeio 2, utilizado en el diagnóstico pre.

I: ¿Coincidiría un venusino en llamarle cuarto menguante a la Luna que vemos?

E: No, porque él no la está mirando desde la Tierra, la vería de otra forma, tal vez vería lo que nosotros vemos oscuro, o tal ve $z$ entera.

I: La parte oscura que vos ponés en la Luna, ¿está producida por qué razón?

E: Porque somos nosotros en cierta forma que tapamos la luz del Sol, los rayos solares van así y esto provoca sombra.

Ivanna, 21 años, post.

$Y$ en algunos casos se sigue utilizando el mismo modelo, sin cambio alguno, que antes de la implementación de la unidad didáctica.

1: Suponé la Luna en cuarto menguante, ¿qué tuvo que haber pasado para que mitad esté en sombra y mitad esté iluminada?

$E: X$, tiene que haber algo que la opaque, o sea que no le permita recibir la luz.

I: ¿Y qué es lo que la opaca?

E: La Tierra.

I.orena, 29 años, post.

\section{CONCLUSIONES}

Este estudio no es cuantitativo, por lo que el comentario que sigue no busca tener peso estadístico, pero sí contextualizar al lector en nuestra realidad.

Consideramos valioso para cualquier proyección educatiya tener en cuenta el número de docentes participantes con relación a nuestra comunidad. Esquel es una ciudad de 23.000 habitantes ubicada en la cordillera sur de la Patagonia argentina, a $2.000 \mathrm{~km}$ de Buenos Aires; su zona de influencia en cuanto a lo social, económico y 
educativo comprende unos $60.000 \mathrm{~km}^{2}$, con una población estimada de 35.000 habitantes, en la que ejercen la docencia de nivel primario aproximadamente unos 500 maestros. Así, estaríamos analizando las ideas existentes en aproximadamente el $15 \%$ de los docentes de nuestra región, lo que nos permite tener una buena evaluación, en principio, de cómo se trabajan estos temas en el nivel primario.

Consideramos que aquí está uno de los factores más importantes de nuestras conclusiones, en lo que se refiere a poder elaborar algún tipo de recomendación para mejorar la educación en ciencias, al menos en nuestra zona.

Este estudio bien podría haber sido hecho con adultos en general y seguramente los modelos y conclusiones podrían haber sido similares. Pero nosotros elegimos trabajar con adultos formados como educadores, actualmente en ejercicio. Es posible pensar que, en la práctica cotidiana, estos maestros pondrán naturatmente en juego el conjunto de ideas que hemos diagnosticado, lo que hará seguramente que los chicos vayan aprendiendo tales ideas y no las que consideramos que debieran aprender. Esto es una especie de «propagación» de las ideas previas, con la gran implicación que tiene debido al efecto multiplicador propio de los maestros, por diversas razones (sistematicidad de la enseñanza, edad de los chicos, autoridad de la figura del maestro, etc.). Esto trae también como consecuencia que los propios chicos tendrán condicionada de algún modo su «libertad» para construir ideas propias en relación con estos temas.

Por esta razón, consideramos de vital importancia, para que la educación en ciencias se desarrolle en forma fructffera desde esta nueva perspectiva constructivista, el rediseño de los profesorados y currículos en ellos utilizados (Driver y Oldham 1986). Se tiende a lograr profundidad conceptual, buscando un cambio de actitud en los docentes y alumnos a través de la vivencia rcal y activa de situaciones diseñadas específicamente desde esta concepción, con un desarrollo de larga duración ya que la modificación de ideas es un proceso dinámico y que requiere tiempo de maduración, y mediante didácticas que deben ser reformuladas en lo general y para cada desarrollo conceptual en lo particular.

Consideramos que luego de nuestra vivencia en estos talleres y del análisis de los diagnósticos pre/post, la respuesta a la pregunta que nos hiciéramos en un comienzo es absolutamente afirmativa.

Estamos convencidos de que ha habido una verdadera evolución desde las ideas previas existentes hacia la estructura conceptual propuesta por nosotros en esta

\section{REFERENCIAS BIBLIOGRÁFICAS}

AULT, C., NOVACK, J. y GOWIN, B., 1988. Constructing Vee maps for clinical interviews on energy concepts. Science Education, Vol. $72(4)$, pp. 515-545. unidad didáctica. Los maestros participantes han adquirido elementos de esta nueva perspectiva conceptual, los cuales se han incorporado de algún modo al conjunto de ideas que ya tenían.

Este estudio permitió vislumbrar esa evolución, aunque no podemos saber qué tan hondo ha calado nuestra propuesta. No es nuestra intención realizar un estudio de seguimiento de estos docentes, pero sí continuaremos trabajando de alguna manera con ellos a través de tres líneas de trabajo:

a) En 1994 comenzará a construirse, en Esquel, eł complejo «Plaza del Cielo: un lugar para aprender y jugar con la astronomía», de nuestra autoría. Todos los elementos que integran este complejo han sido elegidos y diseñados para brindar herramientas didácticas y situaciones experimentales propias de la metodología de cambio conceptual. El citado complejo será una plaza pública de $50 \mathrm{~m} \times 100 \mathrm{~m}$, ubicada en el centro de la ciudad, en la que se dispondrán diversos juegos de plaza adaptados para la enseñanza de la astronomía, y varios elementos como, por ejemplo, un reloj de sol, un globo terráqueo paralelo, un péndulo de Foucault, y representaciones del sistema solar a cscala, tanto en tamaños como en distancias, entre otros. También se construirá un pequeño edificio en el que se instalará un pequeño planetario (GOTO EX3), buscando ser además un centro de intensa actividad educativa y cultural. Este complejo dará a los maestros la posibilidad concreta y permanente de continuar el camino iniciado en su cambio conceptual y a otras personas la posibilidad de comenzarlo, estableciéndose en una acción sistemática, de fácil acceso y sin límite de tiempo.

b) Desde la Plaza del Cielo, y coordinado por el Grupo Esquel de Educación en Ciencias, se tenderá a la conformación de grupos de maestros interesados en abocarse a la investigación y práctica de aquellos conceptos no sólo de astronomía sino de ciencias en general.

c) Finalmente, nos encontramos actualmente escribiendo un libro de texto dirigido fundamentalmente a docentes de nivel primario y secundario, que reflejará lo reportado en este trabajo.

\section{AGRADECIMIENTOS}

A mis compañeros del Grupo Esquel de Educación en Ciencias: Ivanna Ferniot, Beatriz Pérez, Estela Villar, Patricia Martínez, Jorge F'iedrabuena, Marcelo Troiano y Juan Manuel Martinez, ya que las discusiones con ellos mantenidas permitieron mejorar en mucho este trabajo.

A Gabi, Eugenia y Joaquín, mi familia, por el amor que me brindan.

BAXTER, J., 1989. Children's understanding of familiar astronomical events, Science Education, 11 Special Issuc, pp. 502-513. 
BLEGER, J., 1985, Temas de psicología. Cap. Método Clínico. (Ed. Nueva Visión SAI: Buenos Aires).

CAMINO, N. et al., 1991. Estudio de las ideas de estudiantes de nivel terciario en Astronomía (sistema Tierra-Sol-Luna). Memorias de la VII Reunión Nacional de Educación en la Fisica, REF VII. (Mendoza: Argentina).

CAMINO, N., 1993. Un modelo didáctico para evidenciar la causa de las estaciones. Aceptado para su publicación en las Memorias de la Octava Reunión Nacional de Educación en la Física. (Rosario: Argentina).

CAMINO, N., 1993. ¿Cómo es Esquel desde arriba? (Inédito).

DRIVER, R. y EASLEY, J., 1978. Pupils and paradigms: a review of literature related to concept development in adolescent science students, Studies in Science Education, Vol. 5, pp. 61-64.

DRIVER, R., 1981. Pupils alternative frameworks in science, European Journal Science Education, Vol. 3(1), pp. 93-101.

DRIVER, R, 1986, Psicología congnoscitiva y esquemas conceptuales de los alumnos, Enseñanza de las Ciencias, Vol. 4(1), pp. 3-15.

DRIVER, R. y OLDHAM, V., I986. Aconstructivistic approach to curriculum development in science, Studies in Science Education, Vol. 13, pp. 105-122.

DRIVER, R., GUESNE, E. y TIBERGHIEN, A., 1989. Ideas cientificas en la ninez y la adolescencia. (Morata: Madrid).

ERICKSON, G., 1979. Children's conceptions of heat and temperature, Science Education, Vol. 63(2), pp. 221-230.

GIREP, 1986. Cosmos: an educational challenge. (ESA: Copenhague).

GILBERT, J. y WATTS, M., 1983. Concepts, misconceptions and alternative conceptions: changing perspectives in science education, Studies in Science Education, Vol. 10, pp. 61-98.

HEWSON, P. y THORLEY, R, 1989. The conditions of conceptual change in the classroom, International Journal Science Education, Vol. 11 sp. issue, pp. 54]-553.

JONES, B., 1990. «Moon watch, an exercise in observing, recording and communicating». Comunicación personal. (UCE: University of Tasmania).

IONES, B., LINCH, P. y REESINK, C., 1987. Children's conceptions of the Earth, Sun and Moon, Science Education, Vol. 9(1), pp. 43-53.

JONES, B., 1988. «Primary teacher students" explanations of day and night, the seasons and the crescent moon". Paper prepared for presentation to the Conference of the New Zealand Association for Research in Education. (Massey University: Palmerston North, New Zealand).

I,ANCIANO, N., 1989. Sopra l'orizonte: materiali per l'insegnamento e per l'aggiomamento degli insegnanti in Astronomia. (Universitá di Roma «La Sapienzâ: Roma).

LANCIANO, N., 1989. Ver y hablar como Tolomeo y pensar como Copérnico, Enseñanza de las Ciencias, Vol. 7(2), pp. 173-182.

LANCIANO, N., 1992. Dentro il cielo: materiali per l'insegnamento e per l'aggiomamento degli insegnanti in Astronomia. (Universitá dí Roma «La Sapienza»: Roma).
LINDER, C. y ERICKSON, G., 1989. A study of tertiary physics students' conceptualizations of sound, Science Education, I1, Sp. Issue, pp. 491-501.

MOREIRA, M. A. y DOMÍNGUEZ, M. E., 1989. A entrevista clínica como técnica de pesquisa em ensino. Publicación Interior. (UFRGS, Brasil).

NAHOUM, C., 1961. La entrevista psicológica. (Kapelusi: Buenos Aires).

NOVACK, J., 1988. Constructivismo humano: un consenso emergente, Enseñanza de las Ciencias, Vol. 2(3), pp. 213-223.

NUSSBAUM, J. y NOVACK, J., 1976. An assessement of children's concepts of the Earth utilizing structured interviews, Science Education, Vol. 60(4), pp. 535-550.

OSBORNE, R., BELL, B. y GILBERT, J., 1983. Science teaching and children's views of the world, European Journal Science Education, Vol. 5(1), pp. 1-14.

OSBORNE, R. y FREYBERG, P., 1989, El aprendizaje de las ciencias: implicaciones de laciencia de los alumnos. (Narcea: Madrid).

PASACHOFF, J. y PERCY, J. (eds.), 1990, Coll. 105: "The teaching of Astronomy». (Cambridge University Press: Nueva York).

PIAGET, I., 1984. La representación del mundo en el niño. (Morata: Barcelona).

PINES, J., NOVACK, J., POSNER, G. y VANKIRK, J., 1978 The clinical interview: a method for evaluating cognitive structure, Research report 6, Dpt. of Educ., Cornell University.

PFUNDT, H. y DUIT, R, 1991. Students 'alternative frameworks and science education (bibliography), $3^{\mathrm{u}}$ ed. (Kiel: Federat Republic of Germany).

POPE, M. y GILBERT, J., 1983. Personal experience and the construction of knowledge in science, Science Fducation, Vol. 67(2), pp. 193-203.

POSNER, G., STRIKE, K., HEWSON, P. y GERTZOG, W., 1982. Accommodation of a scientific conception: toward a theory of conceptual change, Science Education, Vol. 66(2), pp. 211-227.

POSNER, G. y GERTZOG, W., 1982. The clinical interview and the measurement of conceptual change, Science Education, Vol. 66(2), pp. 195-209.

PROJECT STAR, 1993. The universe in your hands. (Kendall/ Hunt Pub. Co., Estados Unidos).

ROS, R., y CERVANTES, C. (eds.), 1992. Teaching Astronomy: IVth INt. Conference. (Universitat Politècnica de Catalunya, Institut de Ciences de l'Educactó: Barcelona).

SEBASTIÀ, J., 1989. El constructivismo: un marco teórico problemático, Enseñanza de las Ciencias, Vol. 7(2), pp. I58-161

SEQUEIRA, M. y FARIA, M.A., 1989. Concepçoes das crianças sobre o sistema terra-sol-lua, Enseñanza de las Ciencias, número extra (III Congreso) Tomo I, pp. 259-260.

VIGLIETTA, L., 1989. La terra e il suo posto nell'universo. IRRSAE, Scienze, (SEI: Turín).

ZYLBERSTAJN, A., 1985. Comunicación IV Simp. Nac. de Ensino de Fisica. (UFF: Brasil). 